\title{
Reduktion der Überblähung wirkt antientzündlich
}

\begin{abstract}
— Nach den aktuellen GOLD-Leitlinien ist für die Therapie von COPD-Patienten mit mäßiger Symptomatik ein Bronchodilatator vorgesehen. Die POET-Studie mit über 7.300 COPD-Patienten, in der ein langwirksamer Muskarinantagonist (LAMA), z. B. Tiotropium, und ein langwirksamer $\beta$-Agonist (LABA), z. B. Salmeterol, miteinander verglichen wurden, ergab mit einer Reduktion der Exazerbationen um $17 \%$, einen deutlichen Vorteil für Tiotropium. Prof. Andreas Koczulla, Universitätsklinikum Gießen und Marburg, erklärte den Effekt der Bronchodilatation auf die Exazerbationen so: „Bei COPD wird durch die Überblähung Stress auf die Atemwege ausge-
\end{abstract}

übt, und dieser Stress führt zur Produktion von Entzündungs-Mediatoren. Reduziert man die Überblähung, geht auch die Entzündung zurück."

Bei COPD-Patienten mit initial ausgeprägter Symptomatik ist eine LAMA/ LABA-Kombination (z. B. die Fixkombination Aclidiniumbromid/Formoterol 400/12 $\mu$ g, Brimica ${ }^{\oplus}$ Genuair ${ }^{\circledR}$ ) angezeigt. Dies ist deshalb sinnvoll, weil es in den Atemwegen unterschiedliche Rezeptoren gibt. Zum einen sind M3-Rezeptoren zu finden - die Angriffsstelle der LAMA. Über M3-Rezeptoren wird die Aktivität des Parasympathikus gehemmt, der primär den Atemwegstonus des Menschen steuert. Aclidinium besetzt die M3-Re- zeptoren, Acetylcholin kann nicht binden, und die Kontraktion der glatten Bronchialmuskulatur wird verhindert. Der zweite Weg führt über die $\beta_{2-}$ Rezeptoren. LABA bewirken über eine Stimulation dieser Rezeptoren auf der glatten Atemwegsmuskulatur eine Bronchodilatation. „So können Sie additive Effekte erreichen", sagte Koczulla. Wichtig sei dies, weil eine höhere Lungenkapazität dabei helfen kann, den Teufelskreis aus Überblähung, Luftnot, Dekonditionierung und Muskelatrophie zu durchbrechen.

\section{Christina Ott}

- Veranstaltung „Berliner Luft"; Berlin, Mai 2017 (Veranstalter: Berlin-Chemie)

\section{Chronische Rüickenschmerzen}

\section{Die Überempfindlichkeit angehen}

_ Die Chronifizierung von Rückenschmerzen wird maßgeblich durch eine zentrale Sensibilisierung getrieben, die Prof. Ralf Baron, Universitätsklinikum Kiel, und Mitarbeiter in Experimenten objektivierbar darstellen konnten. Dies gab ihnen die Möglichkeit zu prüfen, inwieweit das Ansprechen auf Tapentadol (Palexia $\left.{ }^{\circ}\right)$ durch das Ausmaß der Sensibilisierung beeinflusst wird.

Nach den noch nicht publizierten Daten ist bei Patienten mit einem Sensibilisie-

Chronische Rückenschmerzen beruhen nicht selten auf einer zentralen Sensibilisierung. rungsindex von 0 mit einer etwa 50\%igen Verbesserung der Lebensqualität zu rechnen. „Das ist für einen Patienten mit chronischen Rückenschmerzen schon ein sehr gutes Ergebnis“, betonte der Neurologe. Bei dem höchsten Index von 5 lässt sich sogar eine $80 \%$ ige

Verbesserung erreichen. Der frühzeitige Einsatz von Tapentadol könne eine Schmerzchronifizierung möglicherweise verhindern. Erklärbar seien solche Befunde durch den Wirkmechanismus", mit dem die Substanz der Sensibilisierung an verschie- denen Stellen entgegenwirke. Als $\mu$-Opioidrezeptor-Agonist reduziere es die Aktivität von Schmerzfasern in der Peripherie sowie auf Rückenmarksebene und vermutlich auch im Gehirn. Durch die selektive Noradrenalin-Wiederaufnahmehemmung stabilisiere es außerdem die körpereigene Schmerzkontrolle.

Die Wirksamkeit von Tapentadol unter den Bedingungen der täglichen Praxis belegt eine in Deutschland durchgeführte nicht-interventionelle Studie mit 5.002 Patienten mit starken chronischen Schmerzen [Lange T, Krings D, Waldmann-Rex S. MMW-Fortschr Med. 2015;157(Suppl 4):12-21].

Dr. Matthias Herrmann

-,SChmerzmedizinischer Frühschoppen - Rückenschmerz 360", Deutscher Schmerz- und Palliativtag 2017; Frankfurt/M., März 2017 (Veranstalter: Grünenthal) 